\section{Case Report}

Korean J Transplant 2022;36:67-72 https://doi.org/10.4285/kjt.21.0027
Received October 15, 2021

Revised November 17, 2021

Accepted November 22, 2021

Corresponding author: Taeseung Lee Department of Surgery, Seoul National University Bundang Hospital, Seoul National University College of Medicine, 82 Gumi-ro, 173beon-gil, Bundang-gu, Seongnam 13620, Korea

Tel: +82-31-787-7092

Fax: $+82-31-787-4078$

E-mail: tslee@snubh.org

(C) The Korean Society for Transplantation This is an Open Access article distributed under the terms of the Creative Commons Attribution Non-Commercial License (http://creativecommons.org/licenses/ by-nc/4.0/) which permits unrestricted non-commercial use, distribution, and reproduction in any medium, provided the original work is properly cited.

\section{$\mathrm{KJT}$ \\ KOREAN JOURNAL OF TRANSPLANTATION}

pISSN 2671-8790

elSSN 2671-8804

\title{
The use of polytetrafluoroethylene graft for damaged renal artery in ABO-incompatible living donor kidney transplantation: a case report
}

\author{
Won-Bae Chang ${ }^{1}$, Young-Heun Shin ${ }^{2}$, Hyung Sub Park ${ }^{2}$, Dong-Hwan Kim², \\ Taeseung Lee ${ }^{2}$ \\ ${ }^{1}$ Department of Surgery, Jeju National University Hospital, Jeju National University College of Medicine, Jeju, \\ Korea \\ ${ }^{2}$ Department of Surgery, Seoul National University Bundang Hospital, Seoul National University College of \\ Medicine, Seongnam, Korea
}

Short donor renal vessels during donor nephrectomy represent a technical challenge. The allograft of vessels from deceased donors can be an option for reconstruction; however, cryopreserved vessels are not routinely prepared for living donor kidney transplantation (LDKT). We report a reconstruction of the damaged short renal artery (RA) in LDKT using a polytetrafluoroethylene (PTFE) graft. A 45-year-old male patient underwent ABO-incompatible LDKT from his wife. After donor nephrectomy, we detected a hematoma surrounding the proximal RA of the allograft. The injured segment of the RA was transected, and the short RA was connected to the right external artery of the recipient; however, the blood flow was interrupted by the graft location. Once the arterial anastomosis was removed, the graft was flushed with cold saline, and a PTFE graft was used for the reconstruction of the short RA. Immediate blood flow to the renal graft was excellent without sign of parenchymal infarction until fascial closure. Renal graft Doppler on postoperative day 7 and 3 months showed good blood flow. In this patient, the use of PTFE graft presented no additional morbidity to the kidney transplantation, and no postoperative complications related to its use were noted.

Keywords: Living donor; Kidney transplantation; Renal artery; Polytetrafluoroethylene

\section{INTRODUCTION}

The imbalance of donor organs and recipients has been a longstanding challenge with respect to kidney transplantation [1]. The expanded criteria donor graft has been used increasingly with acceptable outcomes; similarly, the donor from cardiac death is regarded as one of the important sources for grafts, which has comparable results with the donor from brain death [2]. Living donor kidney transplan- tation (LDKT) has become a major treatment option, as living donor grafts have demonstrated similar or better graft survival rates than deceased donors. Furthermore, minimally invasive living donor nephrectomy has been developed with fewer complications [3].

Kidney allografts with variable anatomy or injured vasculature pose a technical challenge to the transplant surgeon [4]. The presence of vascular anomalies, disease of the vessels, or iatrogenic injury to the vasculature during 


\section{HIGHLIGHTS}

- Damaged or short donor renal vessels during donor nephrectomy represent a technical challenge.

- Especially, it is more problematic in living donor kidney transplantation due to the difficulty of securing vascular graft for the reconstruction.

- Polytetrafluoroethylene vascular graft can be used for the reconstruction of damaged renal artery.

organ procurement may necessitate reconstruction or repair of these vessels in order to salvage the allografts for transplantation $[5,6]$. The renal vessels of grafts from deceased donors are usually longer than those from living donors. The renal artery is procured with an aortic patch from a deceased donor, which can be used for artery reconstruction in cases of renal artery injury or short length [7]. On the other hand, the living donor graft does not have an additional vessel graft during nephrectomy. This poses a technical challenge if graft vessels are shortened by anatomical variations or injuries. Stored allograft vessels from deceased donors are useful for the reconstruction of vessels in this situation, but they are not routinely harvested for kidney transplantation. We report a case of kidney transplant in which the organ experienced an iatrogenic injury to the renal artery that occurred during donor nephrectomy. The shortened renal artery was reconstructed using a polytetrafluoroethylene (PTFE) graft, and the recipient had an excellent immediate outcome. To our knowledge, this is the first case report of the use of a PTFE graft to

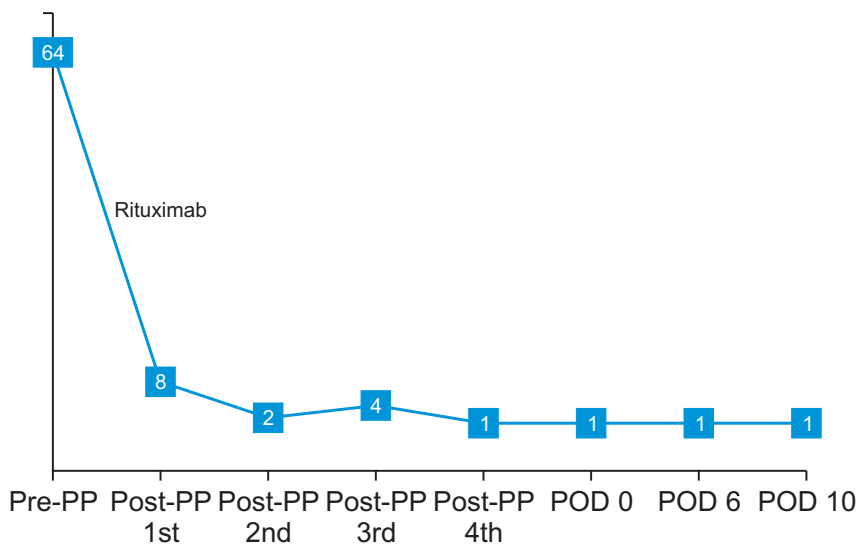

Fig. 1. Anti-A isoagglutinin titers of recipient with plasmapheresis and rituximab administration. PP, plasmapheresis; POD, postoperative day. treat the renal artery in LDKT.

\section{CASE REPORT}

Records and data pertaining to this case were obtained from the patient's medical records at the Seoul National University Bundang Hospital. The Institutional Review Board of Seoul National University Bundang Hospital approved this study (IRB No. B-2022-736-702). Written informed consent was obtained from the patient for the publication of this case report and accompanying images.

A 45-year-old male patient with chronic kidney disease due to immunoglobulin $A$ nephropathy was admitted for preemptive LDKT. His ABO type was $\mathrm{B}+$, and the donor was his wife, a 48-year-old female patient with blood type $A+$. Due to incompatible $A B O$ types between the donor and the recipient, plasmapheresis was performed four times, and rituximab was administered to the recipient preoperatively (Fig. 1). Eight and nine units of fresh frozen plasma were used for the first and second rounds of plasmapheresis, respectively, albumin was administered for the last two rounds of plasmapheresis. Both kidneys had a single artery and vein with no remarkable findings on the donor's preoperative evaluation; therefore, the donor's left kidney was selected for allograft (Fig. 2).

During the operation, the recipient's right iliac fossa was prepared, and the right external iliac artery (EIA) and vein were exposed. Laparoscopic donor nephrectomy

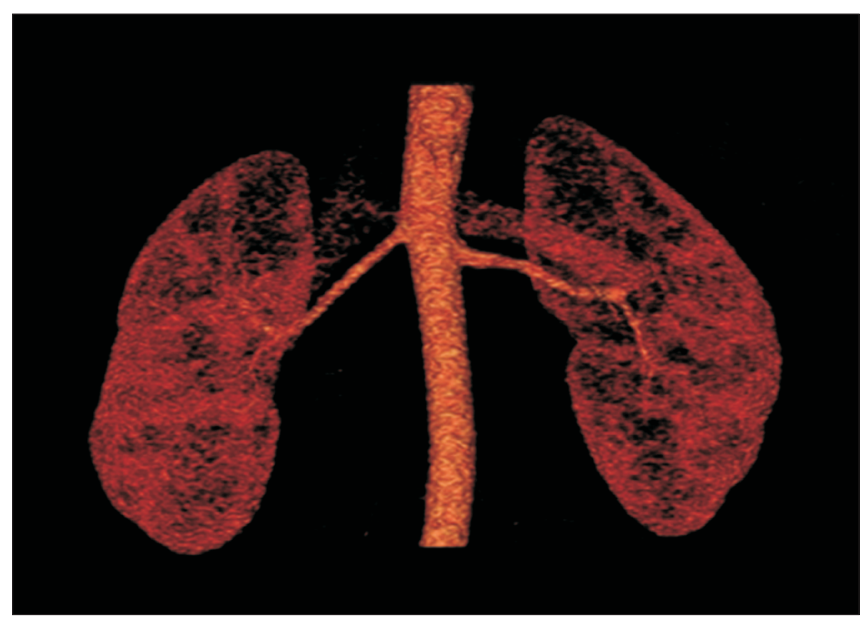

Fig. 2. Three-dimensional computed tomography scan of the donor. A single artery was confirmed in each kidney. 
was performed, and the left kidney graft was delivered to the recipient's operating room. During bench surgery, we located a hematoma around the transected renal artery of the allograft. The initial flush through this renal artery using HTK solution (Custodiol HTK Solution, Durham, NC, USA) was unsuccessful. After careful investigation, we transected the part of the renal artery with the hematoma, and a thrombus in the distal renal artery was removed. The

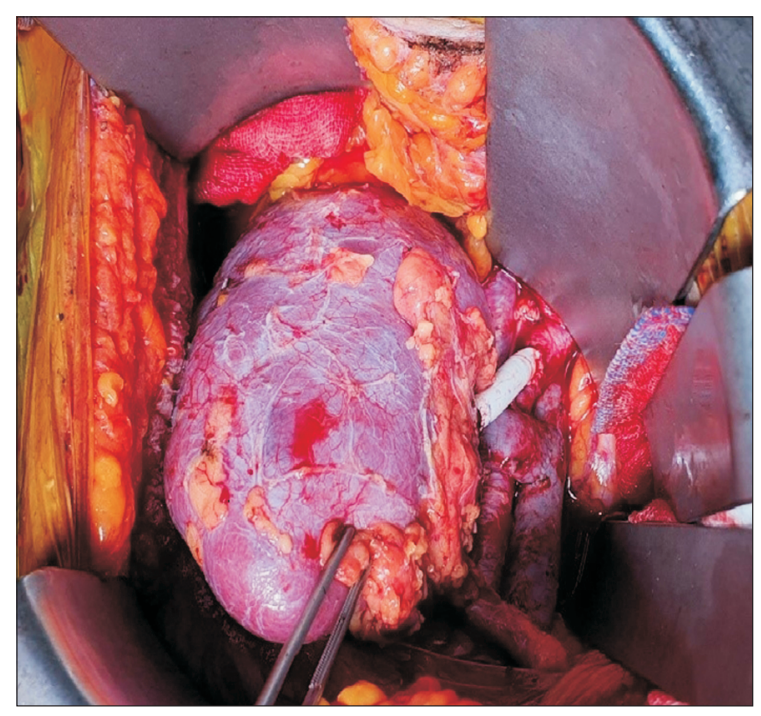

Fig. 3. Short renal artery was reconstructed using polytetrafluoroethylene vascular graft. Renal parenchyma is well perfused after anastomosis. subsequent flush was successfully performed without resistance, and the flow through the renal vein was clear.

The renal artery and vein of the allograft were connected to the right EIA and vein of the recipient, respectively. After vascular anastomosis, the vessels were unclamped and the kidneys were observed to ensure adequate perfusion. During the next step of uretero-cysto anastomosis, we noticed that the color of the kidney had changed to dark purple and was dependent on the graft position. In the natural position, due to gravity, the graft was in the right lateral position, resulting in tension in the renal artery, causing it to fold. As a result of this graft location, the arterial blood flow was significantly reduced.

After careful consideration, we decided to use a synthetic arterial graft. The renal-to-external iliac end-to-side anastomosis was removed after the recipient's EIA was clamped. The kidney graft was flushed with cold saline, and the renal vein was clamped. A $6 \times 40-\mathrm{mm}$ PTFE vascular graft (Impra ePTFE Straight Vascular Grafts; BD, Tempe, AZ, USA) was used to reconstruct the short renal artery. The end of the PTFE anastomosis was joined to the short renal artery of allograft in an end-to-end fashion, while the second end of the graft was connected to the right EIA in an end-to-side fashion (Fig. 3). Systemic heparin was administered during reconstruction. After reconstruction, the vessels were unclamped, confirming excellent blood flow into the kidney, regardless of the kidney location.

Aspirin (100 mg per oral, once a day) and enoxaparin
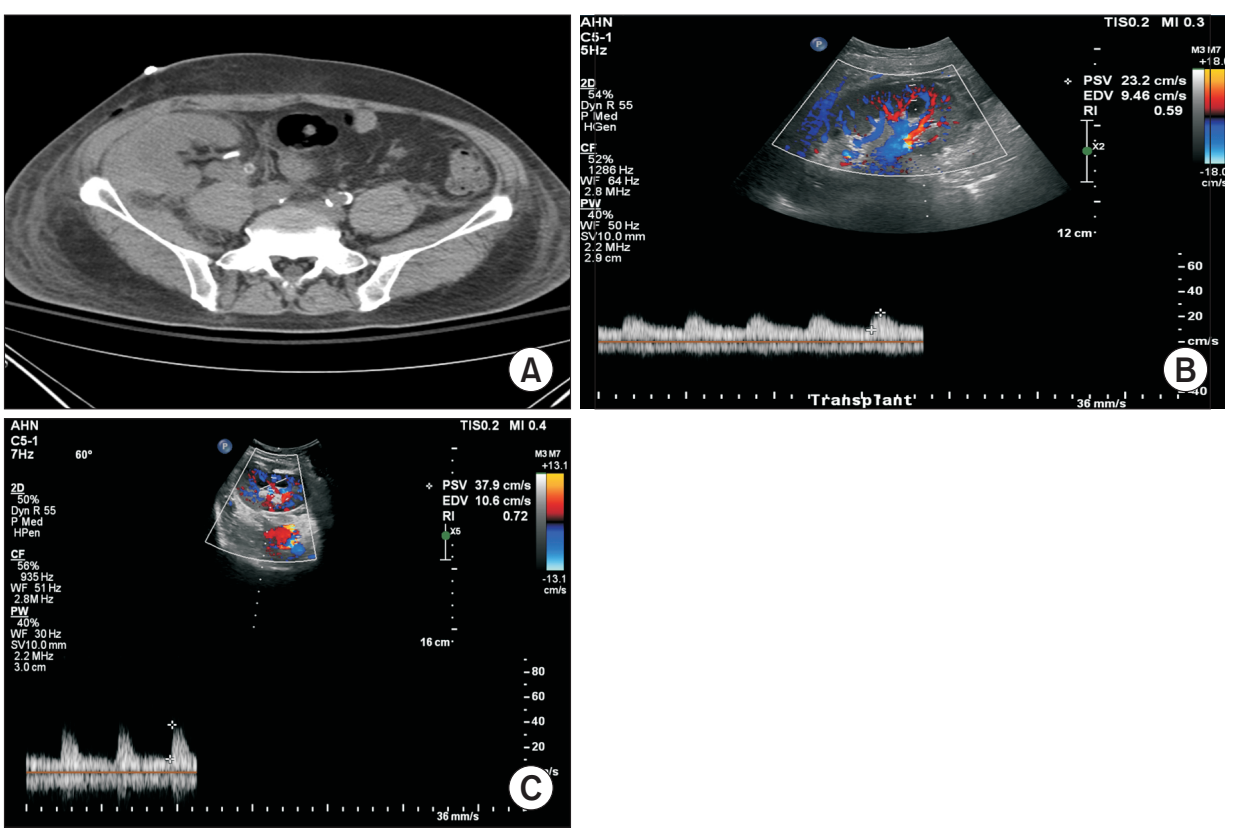

Fig. 4. (A) Non-contrast computed tomography scan on postoperative day (POD) 5 showing mild peri-graft fluid, likely a hematoma. (B) Kidney Doppler on POD 7 showing good vascular flow within the normal range of restrictive index (Rl; 0.59). (C) Follow-up kidney Doppler 3 months after surgery showing good intra- and extra-renal arterial flow within the normal range of RI (0.72). 


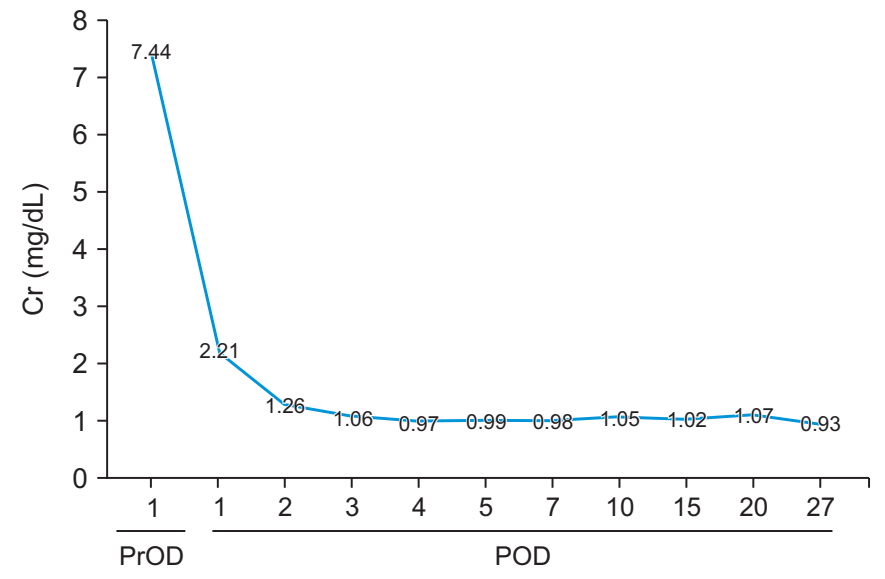

Fig. 5. Pre- and postoperative creatinine $(\mathrm{Cr})$ levels of the recipient. Serum $\mathrm{Cr}$ level reduced in the first 3 days after transplantation and maintained around $1 \mathrm{mg} / \mathrm{dL}$. The last two $\mathrm{Cr}$ values (postoperative day [POD] 20 and 27) were measured in the clinic after discharge. PrOD, preoperative day.

(40 mg, subcutaneously, twice a day) were administered to the recipient from postoperative day (POD) 1 to prevent potential arterial graft occlusion. On POD 3, the patient developed hematuria, and the serum hemoglobin level dropped from $10.2 \mathrm{~g} / \mathrm{dL}$ (POD 1) to $8.7 \mathrm{~g} / \mathrm{dL}$ (POD 3) and then to 6.7 $\mathrm{g} / \mathrm{dL}$ (POD 5). Antiplatelet and anticoagulant agents were discontinued, and non-contrast abdominopelvic computed tomography (CT) showed mild perigraft fluid, which was likely a hematoma (Fig. 4A). We continued holding the aspirin and enoxaparin and gave the recipient ferric carboxymaltose instead of blood transfusion. In the follow-up tests, hemoglobin levels increased up to $7.4 \mathrm{~g} / \mathrm{dL}$ on POD 10 without transfusion. The kidney Doppler test, which was performed on POD 7, showed good vascular flow within the normal range of restrictive index (RI, 0.59) (Fig. 4B). The patient was discharged on POD 17 with a serum hemoglobin level of $9.8 \mathrm{~g} / \mathrm{dL}$ and no other abnormalities. We followed up on the recipient in the clinic on July 13, 2021 (POD 20), during which all other laboratory results were within the normal range. The patient restarted aspirin on POD 20.

\section{DISCUSSION}

Allograft kidneys with complex vascular variation or injuries are frequently declined for transplantation because of the increased risk of complications, such as thrombosis, bleeding, or potentially compromised renal function.
Transplant surgeons face technical challenges in using these organs for transplantation [8]. Due to the shortage of available organs for kidney transplantation, surgeons make every effort to salvage allografts with vascular injuries or complicated vascular variations [7-9]. These efforts of vascular reconstruction with various techniques have achieved results comparable to those achieved using renal allografts without vascular injuries. In a case-control study by McLoughlin et al. [9] renal grafts reconstructed at the bench for injured or variated renal vessels showed equivalent outcomes in terms of graft and patient survival.

In the case of kidney allografts from deceased donors, the renal artery is usually combined with an aortic patch, allowing surgeons to manipulate a longer arterial length for anastomosis with the recipients' iliac arteries. Additionally, these donor aorta patches can be a resource for vascular reconstruction, as Vicéns-Morton et al. [7] reported a reconstruction of the lower polar artery using a tubularized donor aorta patch to replace the injured lower polar artery. If renal vascular injury or abnormality is detected during deceased donor renal procurement, we still have a chance to harvest the donor's vessel for further damaged vascular reconstruction.

On the other hand, renal vessels from living donor kidney allografts are relatively shorter than those from deceased donors; therefore, injury to these vessels often pose a serious challenge. Cryopreserved vascular allografts can be used for renal vascular reconstruction in cases of renal arterial abnormalities in allograft kidneys [10]. However, it is only to be used for cases in which the abnormal vasculature in the donor's kidney is diagnosed before LDKT. Therefore, surgeons should be cautious in preventing graft damage during donor nephrectomy. We retrospectively reviewed our case of donor nephrectomy; however, we could not identify any definite technical problems to explain the hematoma in the proximal renal artery. It is quite probable that the stretching force during renal arterial transection of the donor kidney may have induced the injury and hematoma.

In this case, we initially tried to use the recipient's internal EIA for short graft renal arterial reconstruction, but the length was not sufficient to prevent it from being folded by tension. Using the recipient's great saphenous vein (GSV) for the reconstruction of the damaged renal artery was also considered, but we had to consider the durability of the GSV. Lavigne et al. [11] reported a case in which GSV was used for an aorto-renal bypass in a patient with renal arterial fibromuscular dysplasia who underwent aneurys- 
mal change and subsequently ruptured within 7 years of surgery. Other groups have also reported on the risk of delayed aneurysmal change after using GSV for renal arterial reconstruction [12-14]. One Japanese group reported using the donor's gonadal vein for the reconstruction of multiple renal arteries in living renal transplantation [15]. However, renal arterial reconstruction using a venous graft may have a risk of aneurysmal change, potentially rupturing the graft, as the authors mentioned [11-14]. Therefore, PTFE was used for renal arterial reconstruction with systemic heparin administration.

In this study, aspirin and enoxaparin were used to prevent thrombosis in PTFE grafts. It was initially planned to use enoxaparin for the first 7 days combined with aspirin until aspirin was loaded, followed by aspirin only. However, the recipient had an episode of perinephric hematoma, as confirmed by CT on POD 5, with decreased hemoglobin level; therefore, the treatment plan was changed. Blood products were not administered to the patient as he was a recipient of $A B O$-incompatible transplantation. Therefore, we did not want to increase his chances of producing antibodies that could induce a negative effect on the transplanted kidney. Instead, he was managed conservatively with the administration of prophylactic ferric carboxymaltose and close monitoring. Follow-up kidney Doppler showed good flow into the graft, with a normal RI of 0.59 . Aspirin was resumed on POD 20 and continues to the present.

Few case reports on the use of PTFE grafts for renal vascular reconstruction $[8,16,17]$. Kamel et al. [8] reported the use of PTFE grafts for reconstruction of short or damaged renal arteries in deceased donor kidney transplantation and one case of kidney auto-transplantation. They did not use enoxaparin due to bleeding; however, we considered the risk of graft occlusion and its induced graft failure to be more concerning than the risk of possible bleeding, especially in LDKT. On follow-up in the clinic (POD 20), the patient's creatinine was $1.07 \mathrm{mg} / \mathrm{dL}$ and hemoglobin level was $12.1 \mathrm{~g} / \mathrm{dL}$. The findings of all other laboratory and physical examinations were unremarkable (Fig. 5). Follow-up laboratory tests performed 3 months after surgery also showed all laboratory findings to be within the normal range (creatinine, $1.02 \mathrm{mg} / \mathrm{dL}$; hemoglobin, $15.3 \mathrm{~g} / \mathrm{dL}$ ), with good vascular flow noted on kidney Doppler (Fig. 4C).

Little is known about the long-term outcomes of PTFE vascular grafts. Most of the long-term data on the use of PTFE vascular grafts have been investigated for use in lower limb revascularization $[18,19]$. The lower limb graft is longer and has slower blood flow than do PTFE vascular grafts used for the renal artery. Therefore, grafts for renal arterial reconstruction may result in better outcomes.

Arterial reconstruction using PTFE vascular graft is acceptable for an allograft with Doppler study in LDKT in cases of unexpected renal vascular damage. When available, biologic grafts such as the recipient's internal EIA and cryopreserved vascular graft should be considered. Once the PTFE graft is used, regular follow-up with surveillance Doppler study needs to be performed to detect potential occlusion.

\section{ACKNOWLEDGMENTS}

\section{Conflict of Interest}

No potential conflict of interest relevant to this article was reported.

\section{Funding/Support}

This study was supported by research grant from the Korean Society for Transplantation (2022-00-03001-003).

\section{ORCID}

Won-Bae Chang https://orcid.org/0000-0002-3551-9940 Young-Heun Shin https://orcid.org/0000-0001-5252-2546 Hyung Sub Park https://orcid.org/0000-0001-8748-5925 Dong-Hwan Kim https://orcid.org/0000-0002-2151-0048 Taeseung Lee https://orcid.org/0000-0001-6425-5924

\section{Author Contributions}

Conceptualization: WBC, TL. Methodology: all authors. Formal analysis: WBC, YHS. Writing-original draft: WBC, TL. Writing-review \& editing: WBC, TL.

\section{REFERENCES}

1. Hernández D, Alonso-Titos J, Armas-Padrón AM, Ruiz-Esteban P, Cabello M, López V, et al. Mortality in elderly waiting-list patients versu age-matched kidney transplant recipients: where is the risk? Kidney Blood Pres Res 2018;43:256-75.

2. Ruggenenti P, Silvestre $C$, Boschiero L, Rota G, Furian L, Perna A, et al. Long-term outcome of renal transplantation from octogenarian donors: a multicenter con- 
trolled study. Am J Transplant 2017;17:3159-71.

3. Lee S, Kim J, Shin M, Kim E, Moon J, Jung G, et al. Comparison of outcomes of living and deceased donor kidney grafts surviving longer than 5 years in Korea. Transplant Proc 2010;42:775-7.

4. Ghazanfar A, Tavakoli A, Zaki MR, Pararajasingam R, Campbell T, Parrott NR, et al. The outcomes of living donor renal transplants with multiple renal arteries: a large cohort study with a mean follow-up period of 10 years. Transplant Proc 2010;42:1654-8.

5. De Rosa P, Santangelo M, Ferrara A, Pelosio L, Vallefuoco DM, Caggiano L, et al. Suboptimal kidney: the experience of a single transplant unit. Transplant Proc 2004;36:488-90.

6. Davari HR, Malek-Hossini SA, Salahi H, Bahador A, Rais-Jalali GA, Behzadi S, et al. Sequential anastomosis of accessory renal artery to external iliac artery in the management of renal transplantation with multiple arteries. Transplant Proc 2003;35:329-31.

7. Vicéns-Morton AJ, Callaghan C, Olsburgh J. Reconstruction of a damaged lower polar artery for kidney transplantation using tubularised donor aorta. Case Rep Transplant 2017;2017:3532473.

8. Kamel MH, Thomas AA, Mohan P, Hickey DP. Renal vessel reconstruction in kidney transplantation using a polytetrafluoroethylene (PTFE) vascular graft. Nephrol Dial Transplant 2007;22:1030-2.

9. McLoughlin LC, Davis NF, Dowling CM, Power RE, Mohan P, Hickey DP, et al. Ex vivo reconstruction of the donor renal artery in renal transplantation: a case-control study. Transpl Int 2014;27:458-66.

10. Nguefouet Momo RE, Donato P, Ugolini G, Nacchia F, Mezzetto $L$, Veraldi GF, et al. Kidney transplantation from living donor with monolateral renal artery fibromuscular dysplasia using a cryopreserved iliac graft for arterial reconstruction: a case report and review of the literature. BMC Nephrol 2020;21:451.

11. Lavigne J, Keppenne V, Limet R. Late rupture of a saphenous vein aortorenal graft. J Vasc Surg 1999;29:722-3.

12. Dean RH, Wilson JP, Burko H, Foster JH. Saphenous vein aortorenal bypass grafts: serial arteriographic study. Ann Surg 1974;180:469-78.

13. Travis JA, Hansen KJ, Miller PR, Dean RH, Geary RL. Aneurysmal degeneration and late rupture of an aortorenal vein graft: case report, review of the literature, and implications for conduit selection. J Vasc Surg 2000;32:612-5.

14. Levy MM, Kiang W, Johnson JM, Myers SI. Saphenous vein graft aneurysm with graft-enteric fistula after renal artery bypass. J Vasc Surg 2008;48:738-40.

15. Tomizawa M, Hori S, Nishimura N, Omori C, Nakai $Y$, Miyake $\mathrm{M}$, et al. Arterial reconstruction using the donor's gonadal vein in living renal transplantation with multiple renal arteries: a case report and a literature review. BMC Nephrol 2020;21:190.

16. Blacklock AR, Raabe AL, Lam FT. Renal auto-transplantation with interposed PTFE arterial graft: not necessarily a cure for loin pain/haematuria syndrome. J R Coll Surg Edinb 1999;44:134.

17. Delpín ES. Brief report: successful extension of the transplant renal vein with a synthetic vascular graft. Bol Asoc Med P R 1997;89:206-7.

18. Prager MR, Hoblaj T, Nanobashvili J, Sporn E, Polterauer $\mathrm{P}$, Wagner $\mathrm{O}$, et al. Collagen- versus gelatine-coated Dacron versus stretch PTFE bifurcation grafts for aortoiliac occlusive disease: long-term results of a prospective, randomized multicenter trial. Surgery 2003;134:80-5.

19. Naoum JJ, Arbid EJ. Bypass surgery in limb salvage: polytetrafluoroethylene prosthetic bypass. Methodist Debakey Cardiovasc J 2012;8:43-6. 